\title{
On the search for a $(n, f)$ cross-section reference at intermediate energies
}

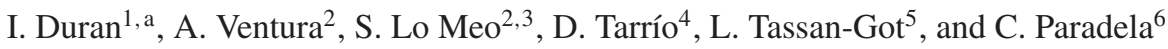 \\ 1 Universidade de Santiago de Compostela (USC), Spain \\ 2 Istituto Nazionale di Fisica Nucleare, Bologna, Italy \\ 3 Agenzia Nazionale per le Nuove Tecnologie, l’Energia e lo Sviluppo Economico Sostenibile (ENEA), Bologna, Italy \\ ${ }^{4}$ Department of Physics and Astronomy, Uppsala University, Sweden \\ 5 Centre National de la Recherche Scientifique/IN2P3 - IPN-Orsay, France \\ ${ }^{6}$ European Commission JRC - IRMM, Geel, Belgium
}

\begin{abstract}
The (n,f) cross-sections proposed as references by the IAEA for ${ }^{235} \mathrm{U},{ }^{238} \mathrm{U}$ and ${ }^{209} \mathrm{Bi}$ are compared with a new analysis that combines the measurements performed at CERN-n_TOF of their cross-section ratios with new calculations done using Monte Carlo codes based on phenomenological models INCL++, GEMINI++, and ABLA07. The calculations are cross-checked with those for the (p,f) reactions, where experimental values are available. We have evaluated in this way the (n,f) cross sections for ${ }^{238} \mathrm{U},{ }^{235} \mathrm{U}$ and ${ }^{209} \mathrm{Bi}$, in the intermediate energy region going from $190 \mathrm{MeV}$ to $2 \mathrm{GeV}$. Our results definitively discard the JENDL/HE-2007 evaluations above $300 \mathrm{MeV}$, falling inside the confidence corridor proposed by IAEA but for the points around $300-400 \mathrm{MeV}$ where a discrepancy is to be noticed.
\end{abstract}

\section{Introduction}

Accurate data on the fission of heavy nuclei at intermediate energies are of a renewed interest for both fundamental and applied nuclear physics. While for the energy range from 20 to $200 \mathrm{MeV}$ there are experimental data good enough to get accurate evaluations, in the energy range from $200 \mathrm{MeV}$ to $1 \mathrm{GeV}$ there are not. The only references come from the evaluated files in the JENDL/HE-2007 nuclear data library that has been seriously criticized by the work of Lo Meo et al. [1]. On the other hand, the IAEA has more recently issued a document [2] on the recommended references to be used in nuclear-fission applications in the intermediate energy region, where the case of ${ }^{235} \mathrm{U},{ }^{238} \mathrm{U},{ }^{209} \mathrm{Bi}$ and ${ }^{\text {nat }} \mathrm{Pb}$ are analysed. The lack of experimental reference points is there clearly stated, since the experimental values of the (p,f) reactions are used even though the conversion of $(\mathrm{p}, \mathrm{f})$ to $(\mathrm{n}, \mathrm{f})$ cross sections remains dependent on theoretical models not accurate enough to properly calibrate the experimental apparatuses used at different laboratories in order to get good fission data.

In this work we discuss the $(\mathrm{n}, \mathrm{f})$ cross-sections proposed as references by the IAEA for ${ }^{235} \mathrm{U},{ }^{238} \mathrm{U}$ and ${ }^{209} \mathrm{Bi}$, comparing them with a new analysis that combines the measurements performed at CERN-n_TOF of their cross-section ratios [3,4] with new calculations done using MC codes [5] based on phenomenological models INCL++, GEMINI++, and ABLA07. The Monte Carlo calculations are cross-checked with the cross sections

a e-mail: Ignacio.duran@usc.es measured for the $(\mathrm{p}, \mathrm{f})$ reactions at higher energies, where experimental values are available.

\section{Cross section calculations}

\subsection{Experimental data}

The data we have used come from experiments performed in different campaigns at the Neutron Time-Of-Flight (n_TOF) facility at CERN [6]. A very intense neutron flux is produced by spallation reactions on a lead target using a $20 \mathrm{GeV} / \mathrm{c}$ proton beam from the CERN Proton Synchrotron (PS). The water surrounding the spallation target acts as a moderator to produce a neutron flux covering a neutron energy range from thermal up to above $1 \mathrm{GeV}$. The long $(185 \mathrm{~m})$ flight path between the spallation target and the experimental area makes it possible to perform high-resolution time-of-flight measurements. More details on the facility and of the neutron beam spectrum can be found in [7]. Fission events were detected using a reaction chamber containing Parallel Plate Avalanche Counters (PPACs) developed at IPN-Orsay. The PPACs used in these experiments have been described previously $[8,9]$.

In this kind of experiments, having several targets measured simultaneously with the same neutron fluence, what one finally gets are the accurate ratios between the number of fissions at each couple of targets. For instance, the ${ }^{209} \mathrm{Bi} /{ }^{235} \mathrm{U}$ ratio is shown in Fig. 1, compared with the one obtained by Laptev et al. [10] up to $200 \mathrm{MeV}$ and by Kotov et al. [11] for the $(\mathrm{p}, \mathrm{f})$ reaction up to $1 \mathrm{GeV}$ (the Prokofiev (p,f) systematics [12] is shown as reference too). 


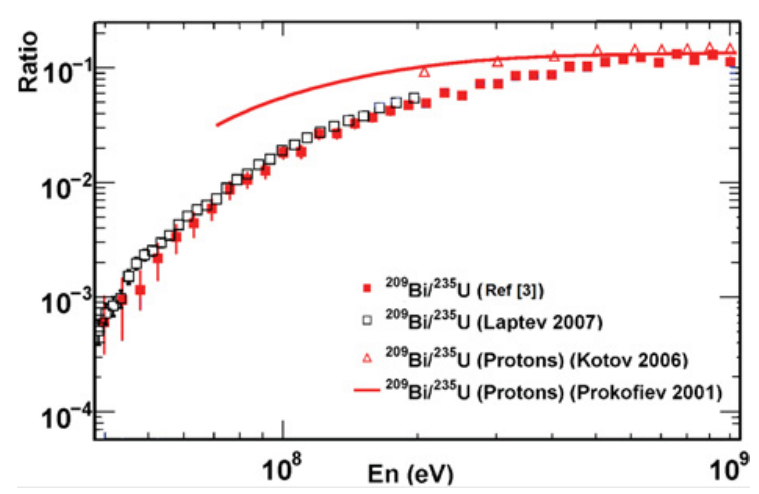

Figure 1. Ratio ${ }^{209} \mathrm{Bi} /{ }^{235} \mathrm{U}$, for $(\mathrm{n}, \mathrm{f})$ and $(\mathrm{p}, \mathrm{f})$ reactions.

In this work we shall deal with the ${ }^{209} \mathrm{Bi} /{ }^{235} \mathrm{U}$, ${ }^{209} \mathrm{Bi} /{ }^{238} \mathrm{U}$, and ${ }^{238} \mathrm{U} /{ }^{235} \mathrm{U}$ cross-section ratios already published in [3] and [4].

\subsection{Monte Carlo calculations}

Fission induced by nucleons at intermediate energies is frequently described as a three-stage process: a fast cascade phase, a pre-equilibrium phase and a final evaporation-fission of equilibrated heavy remnants produced by the fast processes. In the Liège Intranuclear Cascade Model, INCL++, used in Ref. [1] in the energy range from $100 \mathrm{MeV}$ to $1 \mathrm{GeV}$, a self-consistent determination of the stopping time of the fast cascade [13] makes it possible to skip the pre-equilibrium stage, so as to reduce intermediate energy fission to a two stage process. In the Monte Carlo simulation code (Bologna's MC onwards) used in this work in an extended energy range up to $2 \mathrm{GeV}$, version 5.2 [14] of INCL++ was adopted, including multiple pion production [15] that allows, in principle, calculations up to $10-12 \mathrm{GeV}$, while version 5.1.14 adopted in Ref. [1] permitted only single pion production from the delta resonance decays; the versions of the evaporation fission models yielding fission cross sections, GEMINI++ and ABLA07, are those distributed with version 5.2 of INCL++, containing significant changes with respect to the versions adopted in Ref. [1], particularly in ABLA07. The best fits have been obtained, nonetheless, with modest changes in the two adjustable fission parameters that are basic in the decay models: the height of the fission barrier of the remnants, $\mathrm{B}_{f}$, reduced or increased by the same amount, $\Delta \mathrm{B}_{f}$; and either the asymptotic level density parameter, $\mathrm{a}_{f}$, multiplied by a factor $\mathrm{k}$ in ABLA07, or the ratio of $\mathrm{a}_{f}$ to the level density parameter of the neutron channel, $\mathrm{a}_{n}$, in GEMINI++, where the default value is $\mathrm{a}_{f} / \mathrm{a}_{n}=1.036$. Taking advantage of the available $(\mathrm{p}, \mathrm{f})$ experimental data we have adjusted these fission parameters for the three istopes considered in the present work and the results are listed in Table 1.

It is to be pointed out that for the uranium isotopes close numerical results are obtained with both model chains, INCL++/ABLA07 and INCL++/GEMINI++, while for ${ }^{209} \mathrm{Bi}$ only the INCL++/ABLA07 chain yields a satisfactory fit to experimental data below $1 \mathrm{GeV}$. This apparent failure of the INCL++/GEMINI++ chain in the lead-bismuth region was already pointed out in Ref. [1].

Figure 2 compares the results obtained with both versions of the evaporation fission models for the ratio

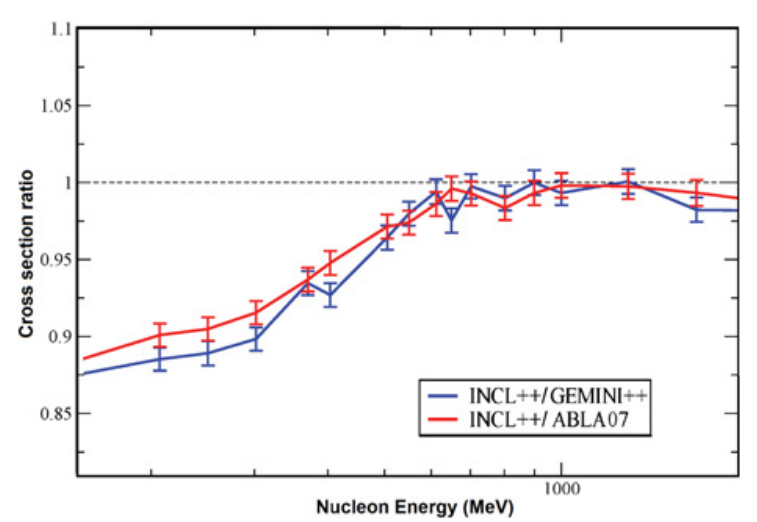

Figure 2. Ratio between (n,f) and (p,f) reactions for ${ }^{238} \mathrm{U}$.

Table 1. Fitting model parameters.

\begin{tabular}{|c|c|c|c|}
\hline Isotope & ${ }^{238} \mathrm{U}$ & ${ }^{235} \mathrm{U}$ & ${ }^{209} \mathrm{Bi}$ \\
\hline $\begin{array}{c}\mathrm{a}_{f} / \mathrm{a}_{n} \\
(\mathrm{GEMINI}++\end{array}$ & 1.039 & 1.045 & - \\
\hline $\begin{array}{c}\Delta \mathrm{B}_{f}(\mathrm{MeV}) \\
(\mathrm{GEMINI++)}\end{array}$ & -0.3 & -0.2 & - \\
\hline $\mathrm{k}(\mathrm{ABLA07})$ & 1.008 & 1.015 & 1.007 \\
\hline $\begin{array}{c}\Delta \mathrm{B}_{f}(\mathrm{MeV}) \\
(\mathrm{ABLA07})\end{array}$ & -0.1 & -0.1 & +0.1 \\
\hline
\end{tabular}

between the $(n, f)$ and $(p, f)$ reactions for ${ }^{238} \mathrm{U}$. It can be seen that this ratio is almost flat and near one from above $500 \mathrm{MeV}$ up to more than $1 \mathrm{GeV}$. This fact will allow us to assign a value to the uncertainty of the $(n, f)$ cross sections calculated around $1 \mathrm{GeV}$, using the experimental $(\mathrm{p}, \mathrm{f})$ cross section values as references.

\subsection{Evaluation procedure}

The following assumptions have been made in this evaluation procedure:

a) The ratio between $(n, f)$ and $(p, f)$ reactions is very close to one around $1 \mathrm{GeV}$.

b) The uncertainties of the $(n, f)$ cross sections at $200 \mathrm{MeV}$ and at $1 \mathrm{GeV}$ are around $2 \%$ as derived from experimental measurements.

c) The $(n, f)$ cross sections show a smooth profile in the whole energy range from $190 \mathrm{MeV}$ up to $2 \mathrm{GeV}$.

d) Both uranium $(\mathrm{n}, \mathrm{f})$ cross sections show a minimum at around $300 \mathrm{MeV}$ and a maximum around $1 \mathrm{GeV}$, as predicted by the Bologna's MC calculations.

Starting from the ratios ${ }^{209} \mathrm{Bi} /{ }^{235} \mathrm{U},{ }^{209} \mathrm{Bi} /{ }^{238} \mathrm{U}$, and ${ }^{238} \mathrm{U} /{ }^{235} \mathrm{U}$ measured at the CERN $-\mathrm{n}_{-}$TOF facility, a smoothing was done by a triangular Bartlett filter, with 25 bins per decade. Then a first order U8 cross section (c.s.) was obtained by multiplying the U8/U5 ratio by the U5 c.s. calculated by the Bologna's MC. This first order U8 c.s. was newly smoothed, using it to get the first order Bi c.s from the Bi/U8 ratio. Next the Bi c.s. values were used to get the U5 ones and once the three first- order c.s. were got, new ratios were obtained and a new loop was performed keeping both values at $200 \mathrm{MeV}$ and $1 \mathrm{GeV}$ always constant. The first one has been taken from the IAEA evaluation and the second one by the fits to the experimental $(\mathrm{p}, \mathrm{f})$ values. 


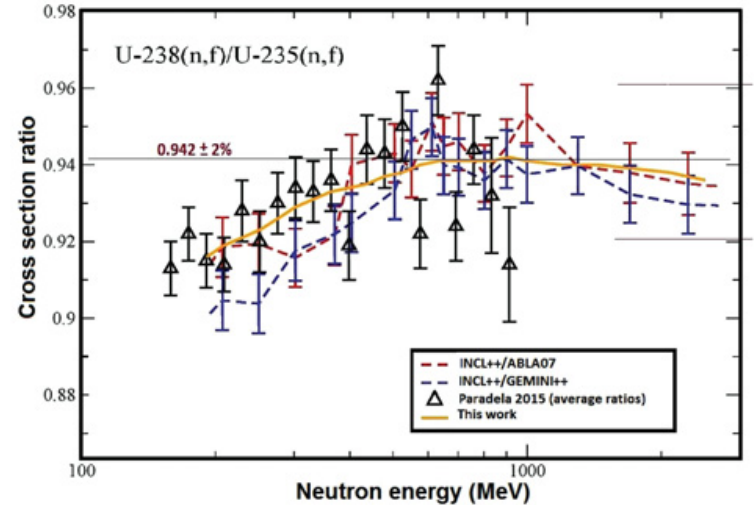

Figure 3. U8(n,f) / U5(n,f) ratio.

Table 2. Evaluated data sets.

\begin{tabular}{lcccccc}
\hline En[MeV] & & & & & & \\
& $\mathrm{U} 5[\mathrm{mb}]$ & $\mathrm{U} 8[\mathrm{mb}]$ & $\mathrm{Bi}[\mathrm{mb}]$ & $\mathrm{Bi} / \mathrm{U} 5$ & $\mathrm{Bi} / \mathrm{U} 8$ & $\mathrm{U} 8 / \mathrm{U} 5$ \\
\hline 191 & $1437(26) 1312(25)$ & $66.8(1.4)$ & 0.0465 & 0.0509 & 0.913 \\
209 & $1430(28)$ & $1311(26)$ & $73.4(1.5)$ & 0.0513 & 0.0560 & 0.917 \\
229 & $1424(30) 1311(27)$ & $81.3(2.0)$ & 0.0571 & 0.0620 & 0.921 \\
251 & $1418(32)$ & $1310(28)$ & $89.8(3.2)$ & 0.0633 & 0.0685 & 0.924 \\
276 & $1418(35)$ & $1313(30)$ & $98.6(4.7)$ & 0.0695 & 0.0751 & 0.926 \\
302 & $1428(40)$ & $1325(35)$ & $107.8(6.0)$ & 0.0755 & 0.0813 & 0.928 \\
331 & $1437(48)$ & $1337(42)$ & $116.7(6.5)$ & 0.0812 & 0.0873 & 0.930 \\
363 & $1447(50)$ & $1349(45)$ & $125.6(7.2)$ & 0.0868 & 0.0931 & 0.932 \\
399 & $1464(50)$ & $1368(45)$ & $134.7(7.8)$ & 0.0920 & 0.0985 & 0.934 \\
437 & $1486(50)$ & $1390(45)$ & $144.6(8.5)$ & 0.0973 & 0.1040 & 0.935 \\
479 & $1512(45)$ & $1417(39)$ & $154.7(8.9)$ & 0.1023 & 0.1092 & 0.937 \\
525 & $1539(42)$ & $1444(36)$ & $164.9(9.0)$ & 0.1071 & 0.1142 & 0.938 \\
576 & $1563(43)$ & $1469(35)$ & $174.9(9.1)$ & 0.1119 & 0.1191 & 0.940 \\
632 & $1586(45)$ & $1491(38)$ & $182.9(8.8)$ & 0.1153 & 0.1227 & 0.940 \\
693 & $1605(42)$ & $1509(36)$ & $188.5(8.9)$ & 0.1174 & 0.1249 & 0.940 \\
759 & $1617(37)$ & $1520(34)$ & $193.0(9.0)$ & 0.1194 & 0.1270 & 0.940 \\
833 & $1622(36)$ & $1525(31)$ & $194.0(9.2)$ & 0.1196 & 0.1272 & 0.940 \\
913 & $1623(35)$ & $1527(30)$ & $193.7(8.9)$ & 0.1193 & 0.1268 & 0.941 \\
1000 & $1624(35)$ & $1529(30)$ & $192.9(8.7)$ & 0.1188 & 0.1262 & 0.942 \\
1200 & $1612(40)$ & $1516(35)$ & $190.2(8.8)$ & 0.1180 & 0.1255 & 0.940 \\
1450 & $1588(40)$ & $1491(40)$ & $185.7(9.6)$ & 0.1169 & 0.1245 & 0.939 \\
1740 & $1566(50)$ & $1468(50)$ & $181.2(9.9)$ & 0.1157 & 0.1234 & 0.937 \\
2090 & $1536(60)$ & $1437(60)$ & $176.8(15.0)$ & 0.1151 & 0.1230 & 0.936 \\
\hline & & & & & & \\
& & & & &
\end{tabular}

\section{Results}

Figure 3 shows the results obtained with this evaluation procedure for the ${ }^{238} \mathrm{U}(\mathrm{n}, \mathrm{f}) /{ }^{235} \mathrm{U}(\mathrm{n}, \mathrm{f})$ ratio. Triangles in black are the experimental points in Ref. [4]; the dotted lines are the Bologna's MC fits and the yellow solid line is the final result of this evaluation. It can be seen that the values of the cross-section ratio around $1 \mathrm{GeV}$, due to the fitting procedure, can be taken with negligible statistical uncertainty, while the systematic one remains within $2 \%$.

Figures 4 and 5 show the (p,f) and (n,f) cross-sections for ${ }^{235} \mathrm{U}$ and ${ }^{238} \mathrm{U}$, respectively. Besides the EXFOR available experimental datasets there are the Bologna's MC fits and the evaluation done in this work. It is worthwhile mentioning the discrepancy found with the IAEA values [2] (here in blue) between $200 \mathrm{MeV}$ and $500 \mathrm{MeV}$.

In Fig. 6 the results for bismuth are plotted, showing for the (p,f) cross sections only the numerical Monte Carlo fit obtained with the INCL++/ABLA07 chain, that gives a very nice agreement with the experimental data below
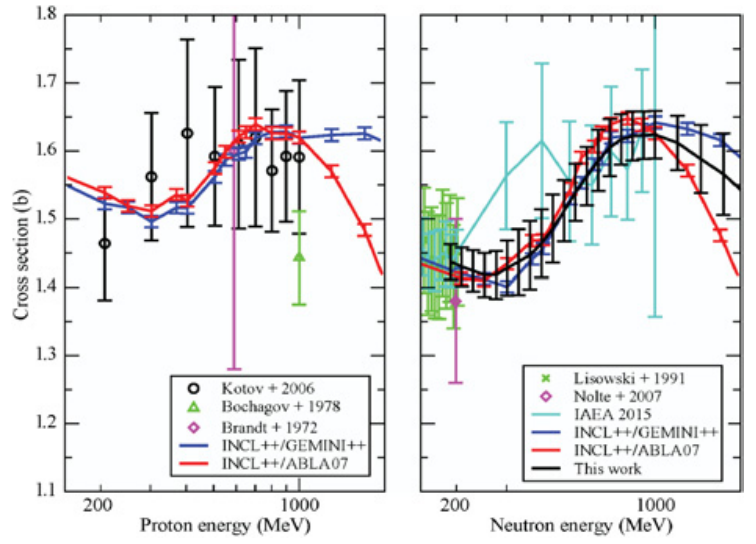

Figure 4. U5(p,f) and U5(n,f) cross sections.
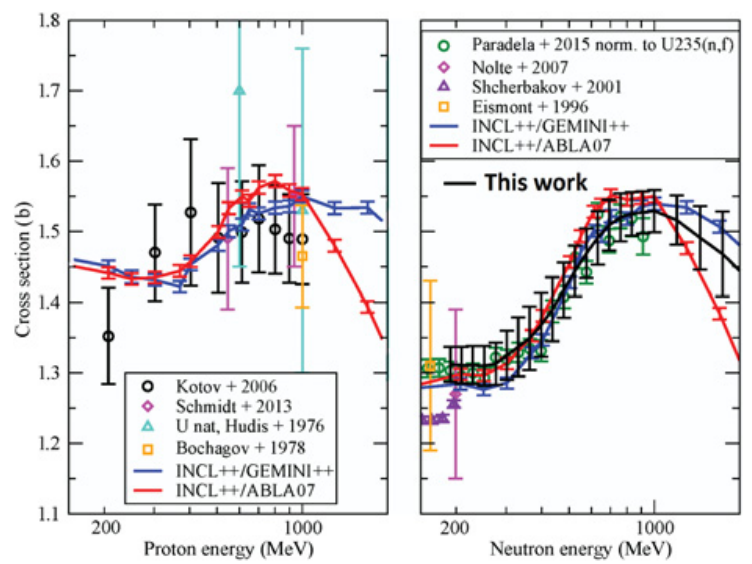

Figure 5. U8(p,f) and U8(n,f) cross sections.
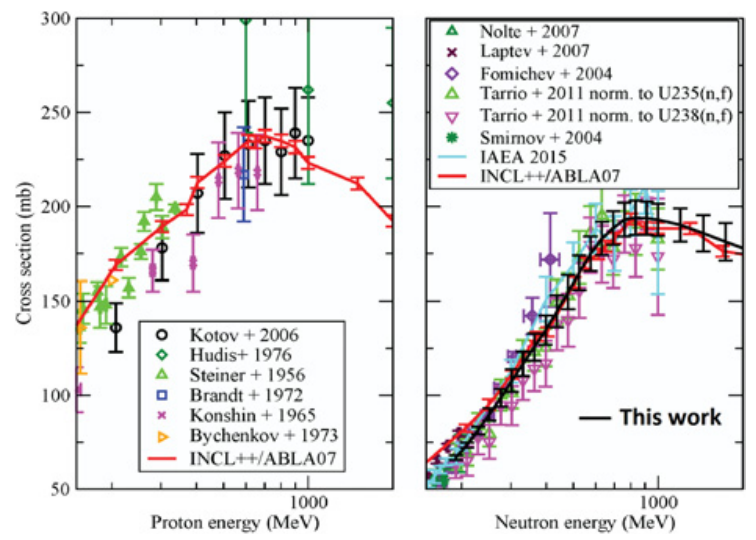

Figure 6. $\mathrm{Bi}(\mathrm{p}, \mathrm{f})$ and $\mathrm{Bi}(\mathrm{n}, \mathrm{f})$ cross sections.

$1 \mathrm{GeV}$, thus defining the fitting model parameters listed in Table 1.

The evaluation done for ${ }^{235} \mathrm{U}$ and ${ }^{238} \mathrm{U}$ is compared with the above mentioned IAEA values as well as with the ones retrieved from JENDL/HE-2007 library, in Fig. 7.

Finally, Table 2 lists the cross sections at different values of the incident neutron energy in the range from $190 \mathrm{MeV}$ up to $2 \mathrm{GeV}$, as they are plotted in Figs. 3-6. The cross section values are given as the point values provided by the fitting functions. 

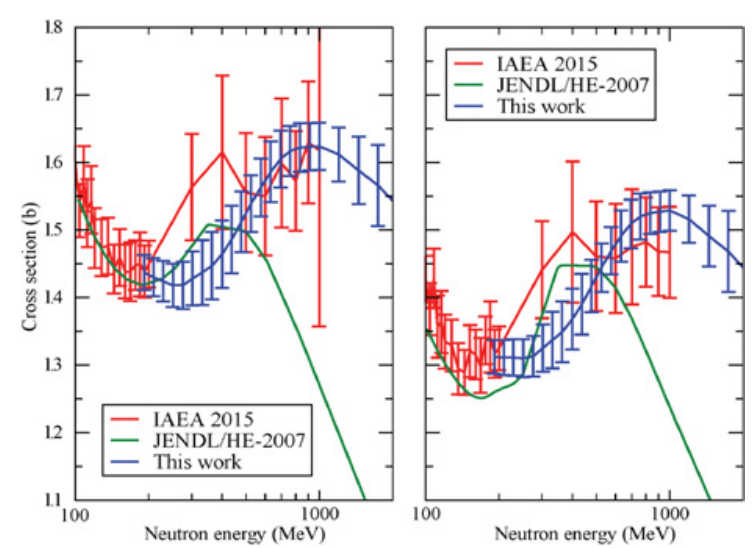

Figure 7. Comparison of evaluations for ${ }^{235} U$ and ${ }^{238} U$.

\section{Conclusions}

The (n,f) cross sections for ${ }^{238} \mathrm{U},{ }^{235} \mathrm{U}$ and ${ }^{209} \mathrm{Bi}$, in the intermediate energy region going from $190 \mathrm{MeV}$ to $2 \mathrm{GeV}$ have been evaluated, giving smooth profiles that can be used as relatively low uncertainty references for further experimental measurements. For these uranium isotopes, our results definitively discard the JENDL/HE-2007 evaluations above $300 \mathrm{MeV}$, falling inside the confidence corridor proposed by IAEA but for the points around $300-400 \mathrm{MeV}$, where a discrepancy is to be noticed. For bismuth, our new parametrization falls inside the uncertainties associated to the IAEA recommended values.
The USC work has been partly supported by the Spanish Agency for Research under grant FPA2013-46236-P.

\section{References}

[1] S. Lo Meo et al. Nucl. Phys. A 933, 43 (2015)

[2] B. Marcinkevicius et al. IAEA report INDC(NDS)$0681(2015)$

[3] D. Tarrio et al. Phys. Rev. C 83, 044620 (2011)

[4] C. Paradela et al. Phys. Rev. C 91, 024602 (2015)

[5] S. Lo Meo et al., paper S087, these Proceedings, submitted to EPJ Web of Conferences (2017)

[6] F. Gunsing et al. Eur. Phys. J. Plus 131, 371 (2016) and references therein

[7] C. Guerrero et al. Eur. Phys. J. A 49, 27 (2013)

[8] C. Paradela et al. Phys. Rev. C 82, 034601 (2010)

[9] D. Tarrio et al. Nucl. Instrum. Methods Phys. Res. A 743, 79 (2014)

[10] A. Laptev et al. "Fast Neutron-Induced Fission of Some Actinides and Sub-Actinides", in Proc. 4th. Intern. Conf. Fission and Properties of Neutron-Rich Nuclei (2008)

[11] A.A. Kotov et al. Phys. Rev. C 74, 034605 (2006)

[12] A.V. Prokofiev, Nucl. Instr. Meth. Phys. Res. A 463, 162 (2001)

[13] A. Boudard et al., Phys. Rev. C 66, 044615 (2002)

[14] D. Mancusi et al., Phys. Rev. C 90, 054602 (2014) 0

[15] S. Pedoux and J. Cugnon, Nucl. Phys. A 866, 16 (2011) 